\title{
Profit Allocation in Fuzzy Cooperative Games in Manufacturing and Logistics Industry
}

\author{
Xiaoyan Wang ${ }^{1}$, Liqi Jiang ${ }^{2}$, LeiShu ${ }^{1}$ \\ ${ }^{1}$ Hefei University (China) \\ ${ }^{2}$ University of International Business \& Economics (China) \\ 1051188719@qq.com,378983993@qq.com,sbulei2009@126.com
}

\section{Abstract:}

Purpose: Alliance between manufacturing and logistics industry is a new model of the joint development of the two industries. A reasonable profit allocation mechanism is the key to ensure the stable operation of the alliance, as well as to achieve the desired objectives. Based on uncertainty of alliance expected return as well as the inherent features of the alliance, this research establishes an improved model of profit allocation in manufacturing and logistics industry alliance.

Design/methodology/approach: This article studies how to introduce comprehensive correction factors to improve interval Shapley value method, which is based on the fact that had been proved by exiting studies. In this study, interval Shapley value method is first applied to calculate the initial allocation of fuzzy cooperative games. Next AHP-GEM method and fuzzy comprehensive evaluation method are incorporated. Based on those results, an improved model of profit allocation is established. After that, a case study is demonstrated the practicality and feasibility of the improved model.

Findings: Profit allocation is a complex issue in fuzzy cooperative games. There're impacts from partner risk sharing, collaborative effort, market competition, innovative contribution as well as resource investment. All these factors should be involved in the profit allocation, and different factors have different weight in importance. 
Practical implications: The new model established in the paper is more scientific and reasonable, and more in line with the actual situation. This method also provides good incentives to each enterprise to ensure the healthy and stable development of the alliance.

Originality/value: Based on alliance characteristics, this paper establishes an indicator system and a new model for profit allocation in manufacturing and logistics industry alliance, using AHP-GEM method.

Keywords: manufacturing and logistics industry, fuzzy cooperative games, improved interval Shapley value, AHP-GEM method, profit allocation

\section{Introduction}

In recent years, the joint development of manufacturing and logistics industry has attracted widespread attention of the domestic and foreign scholars. Existing studies are mainly focused on the theoretical explanation of the cooperation between manufacturing and logistics industry, two industry joint development model, joint development strategy as well as the joint development empirical analysis, etc. Studies on profit allocation between manufacturing and logistics industry are very rare. Existing study on manufacturing and logistics industry profit allocation is mainly about pricing on logistics outsourcing. For example, Ni (2007) established the logistics outsourcing payoff matrix. Using game theory, Luo and Mao (2010), established pricing game model of logistics outsourcing for both sides, and provided the equilibrium solution. Chen and Li (2011) designed logistics outsourcing profit sharing incentive contract based on specific investment. Manufacturing and logistics industry alliance is constraint by agreements, compliances based on common interests. Members collaborate with each other under the promise of information sharing. As a result, the alliance can be a cooperative game. Profit allocation based on logistics outsourcing will only intensify competition between the two industries. Therefore, this method does not solve the profit allocation problem in manufacturing and logistics industry cooperative games. Shapley Value method is widely applied to solve the $n$-person cooperative game cooperative gains allocation.

Cooperative game can be divided into two categories (Meng \& Zhang, 2011): the classical cooperative game and fuzzy cooperative game. In reality, because of the complexity of the cooperation process, the uncertainty of decision-making environment, the ambiguity of problem understanding, and the incompleteness of information, n-person game theory research in fuzzy environment has become a hot topic in current game theory research. Fuzzy cooperative game research mainly focuses on two aspects (Tan, 2011): the research of $n$ person cooperative game with fuzzy coalition; the research of $n$-person cooperative game with fuzzy coalition value. 
The core issue in any cooperative game is about cooperative profit allocation. Considering alliance profit uncertainty due to the partner's fuzzy preference, Mares (2001) explored fuzzy returns, obtained the Shapley value of fuzzy membership function. Surajit (2008) studied cooperative games with fuzzy coalition and fuzzy payment from fuzzy alliance perspective. The cooperative game with interval payment is a special kind of fuzzy cooperative game. Chen and Zhang (2006) used fuzzy math theory proposed a fuzzy Shapley value that can meet validity, symmetry and additive requirements. Yu and Zhang (2008) analyzed the circumstances when the cooperative game payoff function is an interval value, he also proposed the Shapley value with interval pay. Through establishment of an axiomatic system, Tan (2010) studied Shapley value with interval payment under universal condition.

This article studies how to introduce comprehensive correction factors to improve interval Shapley value method, which is based on the fact that had been proved by exiting studies that there's uncertainty of alliance expected return as well as the inherent features of the manufacturing and logistics industry. The article also establishes an improved model of profit allocation in fuzzy cooperative games in manufacturing and logistics industry. Also, the article takes manufacturing and logistics alliance as example, demonstrates the practicality and feasibility of the improved model.

\section{The Introduction of the Interval Payoff Cooperation Game and the Interval Shapley Value Method}

Two-tuples $(N, \bar{V})$ is called the fuzzy cooperation game of the players set $N=\{1,2, \ldots n\}$, and $\bar{V}$ is the fuzzy payoff function which is defined on the power set $P(N)$ of $N$, and values in the interval set $\bar{R}$, that means $\bar{V}: P(N) \rightarrow \bar{R}$, and $\bar{V}(\varnothing)=0$. The interval payoff function $\bar{V}(S)=\left[V^{-}\right.$ $(S), V^{+}(S)$ ] means that the maximum profit interval which each player can obtain through cooperation of the coalition $S \in P(N)$. Interval fuzzy cooperation game set is marked as $G_{I}(N)$ (Yu \& Zhang, 2008). Here, suppose $\forall S \subseteq N, \bar{V}(S) 0$.

- Definition 1: For the cooperation game $\mathrm{G}_{\mathrm{I}}(\mathrm{N})$ with the interval payoff, if $\forall T \in P(N), S \in P(N)$ all conform with (meet the condition) $\bar{V}(T \cap S)=\bar{V}(T)$, then $S$ can be called the carrier.

- Definition 2: Suppose $N=\{1,2, \ldots n\}$, for any $\bar{v} \in G_{I}(N)$, the Shapley value with interval coalition value $n$-persons games $\bar{V}$ is a vector function $\bar{\varphi}: \mathrm{G}_{\mathrm{I}}(\mathrm{N}) \rightarrow(\overline{\mathrm{R}})^{\mathrm{nP}(\mathrm{N})}$ which conforms with following system of axioms, and $\bar{\varphi}=\left(\bar{\varphi}_{1}, \ldots, \bar{\varphi}_{\mathrm{n}}\right)$.

Axiom 1 (interval validity): for any carrier $\mathbf{S}$ of $\overline{\mathrm{V}}$, then $\sum_{i \in S} \bar{\varphi}(\overline{\mathrm{V}})=\bar{V}(\mathrm{~S})$.

Axiom2 (interval symmetry): for any permutation $\pi$ and $i \in N$ then $\bar{\varphi}_{\pi(i)}(\bar{V})=\bar{\varphi}_{i}(\bar{V})$. 
Axiom3 (interval superposition): for any two cooperation games $\bar{V} 1, \bar{V} 2 \in G_{1}(N)$ then $\overline{\varphi_{i}}(\overline{\mathrm{V}} 1+\overline{\mathrm{V}} 2)=\bar{\varphi}_{i}(\overline{\mathrm{V}} 1)+\bar{\varphi}_{i}(\overline{\mathrm{V} 2}), \forall \mathrm{i} \in \mathrm{N}$.

Proposition 1: The defined vector function $\bar{\varphi}: \mathrm{G}_{1}(\mathrm{~N}) \rightarrow\left(\overline{\mathrm{R}}^{\mathrm{n}}\right)^{\mathrm{P}(\mathrm{N})}, \bar{\varphi}(\overline{\mathrm{V}})=\left(\bar{\varphi}_{1}(\overline{\mathrm{V}}), \ldots, \overline{\varphi_{\mathrm{N}}}(\overline{\mathrm{V}})\right)$, $\overline{\varphi_{1}}(\overline{\mathrm{V}})=\sum_{\mathrm{i} \in \mathrm{S} \in \mathrm{p}(\mathrm{N})} \gamma S(\overline{\mathrm{V}}(\mathrm{S}) \ominus \overline{\mathrm{V}}(\mathrm{S} \backslash\{\mathrm{i}\}))$ is the vector of the Shapley value of the game $\overline{\mathrm{V}}$. In the formula $\gamma s=\frac{(s-1) !(n-s) !}{n !}$, and $s$ is the person number of the coalition $s, n$ is the player number of the game, $\forall i \in N$,

$$
\varphi^{+}(\bar{V})=\sum_{i \in S \in \mathrm{p}(\mathrm{N})} \gamma S\left(V^{+}(S)-V^{+}(S \backslash\{i\})\right), \varphi_{i}(\bar{V})=\sum_{i \in S \in \mathrm{p}(\mathrm{N})} \gamma S(V(S)-V(S \backslash\{i\}))
$$

The proof process can be seen in the reference (Tan, 2010).Here, a new interval number subtraction (Tan, 2010) $\Theta: \bar{x} \ominus \bar{y}=\left[x^{-}-y^{-}, x^{+}-y^{+}\right]$is defined, which can be regarded as a inverse operation of interval number addition. For any a $>0$ positive number, $\bar{x} \ominus \bar{y} \in \bar{R}$, then $a(\bar{x} \ominus \bar{y})=a \bar{x} \ominus a \bar{y}$.It should be noted that if this method is taken, $\bar{x}-\bar{y} \quad x^{+}-y^{-}$may be possible.

- Definition 3: For strategy $(N, \bar{V})$, if the vector function $\bar{\varphi}: G_{1}(N) \rightarrow\left(\bar{R}^{n}\right)^{P(N)}$ and $\bar{\varphi}=\left(\bar{\varphi}_{1}, \ldots, \bar{\varphi}_{n}\right)$ is called an allocation of cooperation games with interval payment, then the following requirements should be met:

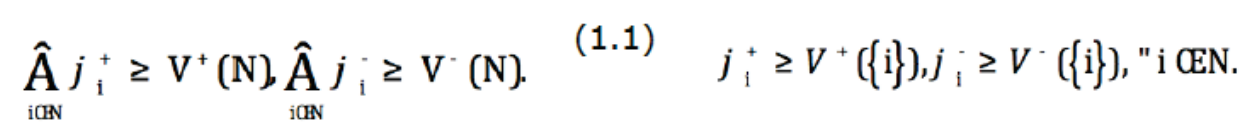

Therefore, it is easy to prove that the interval Shapley value is a natural fuzzy continuation of the Shapley value of the classic cooperation game, and it is more realistic to reality to use interval number to represent the payoff function (Tan, 2010).

\section{Improved Interval Shapley Value Method for Profit Allocation of the Manufacturing and Logistics Industry Fuzzy Cooperative Games}

Interval Shapley value method is able to reflect the overall contribution as well as the importance of the collaborators in cooperation. It also provides a good reference for the uncertainty cooperation allocation of the income. However, this method fails to subdivide the contribution factors, nor weights differently among different factors. It is simply reflects the fuzzy contribution from the collaborators. In the joint development of manufacture and logistics industry, risk sharing, collaborative efforts, market competition, innovative 
contribution and resource investment affect the profit allocation. All these factors should be involved in the profit allocation, and different factors have different weight in importance. Therefore, it is necessary to improve the interval Shapley Value method.

This paper first carries out preliminary profit allocation in manufacturing and logistics industry fuzzy cooperative games based on Interval Shapley Value method in proposition 1, the result of this exercise is then used as one of the evaluation factors for the final profit allocation. Next, the weights of each profit allocation factors are determined using AHP-GEM (Analytic Hierarchy Process and Group Eigenvalue Method) (Qiu, 1997). Furthermore, the profit allocation impact factor is calculated with fuzzy comprehensive evaluation method for each partner. By introducing the correction factor, the paper establishes improved model of the profit allocation for manufacturing and logistics industry fuzzy cooperative games.

\subsection{Determine Profit Allocation Evaluation Factor and its Weight}

Here are the steps to determine profit allocation evaluation factor and its weight in fuzzy cooperative games using AHP-GEM (Qiu, 1997).

\subsubsection{Select Evaluation Factors and Create a Hierarchical Structure}

Based on the nature of each alliance partner, select evaluation factors, and construct a hierarchical model of profit allocation indicator index, including Goal Layer, Criteria Layer and Indicator Layer.

\subsubsection{Construct Subject Expert Evaluation Matrix}

Based on the hierarchical structure, construct an evaluation matrix for the Criteria Layer and a diode matrix for Indicator Layer based on Criteria Layer. The method for constructing evaluation matrix is as follows: $m$ experts from expert group $G$ provide evaluations of $n$ indicators to construct the order matrix $m \times n$, as follows $\mathrm{X}=\left[\mathrm{x}_{\alpha \beta}\right]_{m \times n}$, in which, $\mathrm{X}_{\alpha \beta} \in[\alpha, \beta](\alpha=$ $1,2 \ldots, \mathrm{m} ; \beta=1,2, \ldots, \mathrm{n})$ is the evaluation given by the expert to the indicator $\beta$.

Determine Relative Weight. Based on the calculation of single weight for the indicators in Criteria Layer, normalize to get the relative weights of evaluation indicators. The calculation process is: Raise a transposed evaluation matrix $\mathrm{X}$ to get $\mathrm{F}$, that is $\mathrm{F}=\mathrm{X}^{\top} X$, the corresponding eigenvector of the max eigenvalue of $\mathrm{F}$ is the optimal decision $\mathrm{X}^{*}$. Above process is based on the construction of an ideal expert model. 
Definition of an ideal expert: an expert that has the smallest sum of angles between his scoring vector and the scoring vectors of the other experts in a group is called the ideal or optimal expert of the group.

An ideal expert model: Based on the definition of an ideal expert, the optimal decision $\mathrm{X}^{*}$ is the vector for the formula $f=\sum_{i=1}^{m}\left(b^{\top} x_{i}\right)^{2}$ at a max value. In the formula, $\forall b=\left(b_{1}, b_{2}, \ldots, b_{n}\right) \in E_{n}$, mean $\forall \mathrm{b} \in \mathrm{E}_{\mathrm{n}}, \max _{\mathrm{b} \in \mathrm{E}^{\mathrm{X}}} \sum_{\mathrm{i}=1}^{\mathrm{m}}\left(\mathrm{b}^{\top} \mathrm{X}_{\mathrm{i}}\right)^{2}=\rho_{\max }, \rho_{\max }$ is the max eigenvalue of the matrix $\mathrm{F}=\mathrm{X}^{\top} X, \mathrm{X}^{*}$ is corresponding eigenvector $\mathrm{X}^{\top} X$ of $\rho_{\max }$ and $\left|\mathrm{X}^{*}\right|=1$.

Solution for the ideal expert model: There're two common solutions out of the many solutions to the ideal expert model. One is to determine accuracy requirement, and then to run iteration calculation of the matrix $\mathrm{F}$ using the power method in numerical algebra to get $\mathrm{X}^{*}$. The other is to use software to calculate the max positive eigenvalue $\rho_{\max }$ of the matrix $F_{\text {, then }} \mathrm{X}^{*}$ is the positive eigenvector that $\rho_{\max }$ is corresponding to $\mathrm{F}$.

\subsubsection{Determine Combined Weight of Indicator Layer Relative to the Overall Goal Layer}

The weights of each indicator in the Indicator Layer, $C_{i j}(i=1,2, \ldots, k, j=1,2, \ldots, t)$, to the Goal Layer is the product of the indicator weight and its weight in the Criteria Layer. Which is $w_{i j}=w_{i}$. $w_{j}^{i}, w_{i}$ is the weight of the indicator $C_{i}$ in the Criteria Layer to the Goal Layer, $w_{j}^{i}$ is the weight of the indicator $C_{i j}$ in the Indicator Layer to the indicator in the Criteria Layer. The weight vector of each indicator for Indicator Layer of the alliance profit allocation is $W=\left[W_{1}, \ldots, W_{i}, \ldots, W_{k}\right]$. When $\mathrm{w}_{\mathrm{i}} \in[0,1]$ and $\sum_{\mathrm{i}=1}^{\mathrm{k}} \mathrm{w}_{\mathrm{i}}=1$, the combination weight vector for the Indicator Layer is $W_{i}=\left[w_{i 1}, \ldots, w_{i j}, \ldots, w_{i t}\right], w_{i j} \in[0,1]$, and $\sum w_{i j}=1$. When $W_{i}^{\prime}=w_{1}^{i}, \ldots, w_{j}^{i}, \ldots, w_{t}^{i}, \sum_{j=1}^{t} w_{j}^{i}=1$.

Clearly, the combination of AHP and GEM methods keeps the process of scientific analysis in the hierarchical structure of AHP, while avoiding the inconsistencies in the construction of Saaty Matrix, making multi-criteria decision-making more scientific and accurate. 


\subsection{Determine the Profit Allocation Comprehensive Impact Factors}

Here are the three steps to establish profit allocation comprehensive impact factors using fuzzy comprehensive evaluation method.

- Establish review set V. The review set is divided into five levels, which is $\mathrm{V}=\left(\mathrm{V}_{1}, \mathrm{~V}_{2}, \mathrm{~V}_{3}, \mathrm{~V}_{4}, \mathrm{~V}_{5}\right)=$ (High, medium high, medium, medium low, low $)=(0.9,0.7,0.5$, 0.3. 0.1).

- Develop membership subset. Have subject experts evaluate the various factors referring to review set to get fuzzy subset $\mathrm{R}_{\mathrm{i}}=\left(\mathrm{r}_{\mathrm{i} 1}, \ldots, \mathrm{r}_{\mathrm{ij}}, \ldots, \mathrm{r}_{\mathrm{i} 5}\right) . R_{\mathrm{i}}$ refers to the membership of the $\mathrm{i}$-th indicators in the corresponding reviews set, which is $r_{i j}=$ number of people on the $\mathrm{i}$-th indicator choose $\mathrm{V}_{\mathrm{i}}$ level / number of all people participate. Fuzzy evaluation matrix $R$ is calculated after fuzzy transformation.

- Finally, apply fuzzy arithmetic on the fuzzy evaluation matrix and factors weight vector sets $W$, get fuzzy comprehensive evaluation result sets $B, B=W \Theta R$. Then using fuzzy allocation method to normalize the indicators, gets $B^{\prime}=(b 1, b 2, b 3, b 4, b 5)$. Next, the impact factor of the profit allocation of manufacturing and logistics industry alliance is $\mathrm{P}_{\mathrm{i}}=\mathrm{B}^{\prime} \mathrm{V}^{\top}$, and also is normalized to get $\mathrm{P}^{\prime}{ }_{\mathrm{i}}$.

\subsection{Improved Interval Shapley Value Method with the Correction Factor}

Without considering the risk sharing, collaborative effort, market competition, innovative contribution and resource investment, in the manufacturing and logistics industry alliance that composed of enterprises, each enterprise has the same impact on the profit allocation, which is $1 / n$. From above calculation, cooperative enterprise $i$ has comprehensive impact factor $P^{\prime}{ }_{i}$, the variance is $\Delta \mathrm{P}_{i}=\mathrm{P}_{i}^{\prime}-\frac{1}{n}$, then $\sum_{i=1}^{n} \mathrm{P}_{i}^{\prime}=1$ and $\sum_{i=1}^{n} \Delta \mathrm{P}_{\mathrm{i}}=0, \Delta \mathrm{P}_{\mathrm{i}}$ is the impact factor variance between actual situation and theoretical model, which is correction factor of profit allocation of alliance. 
Suppose there's a manufacturing and logistics industry alliance that composed of $n$ enterprises. The total profit interval is $\bar{V}(S)=\left[V(S), V^{+}(S)\right]$, then enterprise actual updated profit allocation is $\Delta \varphi_{i}{ }^{\prime}(\bar{V})=\Delta \mathrm{P}_{i} \times \bar{V}(\mathrm{~S}), \Delta \varphi_{i}{ }^{\prime}(\bar{V})$ can be positive or negative. Enterprise i actual profit interval is $\varphi_{\mathrm{\imath}}{ }^{\prime}(\bar{V})=\varphi_{\mathrm{\imath}}{ }^{\prime}(\bar{V})+\Delta \varphi_{\mathrm{i}}{ }^{\prime}(\bar{V})$.

$$
\varphi_{\mathrm{i}}^{\prime}(\bar{V})=\left[\varphi_{\mathrm{i}}^{-}(\bar{V})^{\prime}, \varphi_{\mathrm{i}}^{+}(\bar{V})^{\prime}\right]=\left[\varphi_{\mathrm{i}}^{-}(\bar{V})+\Delta \varphi_{\mathrm{i}}^{-}(\bar{V}), \varphi_{\mathrm{i}}^{+}(\bar{V})+\Delta \varphi_{\mathrm{i}}^{+}(\bar{V})\right]
$$

Because $\sum_{i=1}^{\mathrm{n}} \Delta \varphi_{i}{ }^{\prime}(\overline{\mathrm{V}})=\sum_{i=1}^{\mathrm{n}}\left(\Delta \mathrm{P}_{\mathrm{i}} \times \overline{\mathrm{V}}(\mathrm{S})\right)=\sum_{i=1}^{\mathrm{n}}\left(\Delta \mathrm{P}_{\mathrm{i}}\right) \times \overline{\mathrm{V}}(\mathrm{S})=0$

Therefore $\sum_{i=1}^{\mathrm{n}} \varphi_{i}{ }^{\prime}(\overline{\mathrm{V}})=\sum_{i=1}^{\mathrm{n}} \varphi_{i}(\overline{\mathrm{V}})+\sum_{i=1}^{\mathrm{n}} \Delta \varphi_{i}{ }^{\prime}(\overline{\mathrm{V}})=\sum_{i=1}^{\mathrm{n}} \varphi_{i}(\overline{\mathrm{V}})=\overline{\mathrm{V}}(\mathrm{S})$

The interval Shapley Value method with comprehensive correction factor is more scientific and reasonable, and more in line with the actual situation. This method facilitates overall coordination of the alliance, enhances incentive system among alliance members, and hence ensures the stability of the alliance.

\section{Case Study}

\subsection{Case Description}

Suppose there's an alliance comprises one manufacturing enterprise $A$ and two logistics enterprises B and C. B, C provides specialized logistics services to A, and the logistics services are the same. This alliance has taken the project-oriented operation; all three parties have resources invested in the projects. Suppose A, B and C are rational economic men, who share full information with maximizing profits as the goal. The profit interval of a cooperation project is shown in Table 1.

\begin{tabular}{|c|c|c|c|c|c|c|c|}
\hline alliance & $\mathbf{A}$ & $\mathbf{B}$ & $\mathbf{C}$ & $\{\mathbf{A}, \mathbf{B}\}$ & $\{\mathbf{B}, \mathbf{C}\}$ & $\{\mathbf{A}, \mathbf{C}\}$ & $\{\mathbf{A}, \mathbf{B}, \mathbf{C}\}$ \\
\hline $\begin{array}{c}\text { profit } \\
\text { interval }\end{array}$ & {$[10,14]$} & {$[12,14]$} & {$[12,16]$} & {$[32,40]$} & {$[26,36]$} & {$[38,44]$} & {$[54,60]$} \\
\hline
\end{tabular}

Table 1. Profit intervals of various A, B, C alliance

\subsection{Pre-improved Profit Allocation with Shapley Interval Value Method}

Interval Shapley value is calculated using formula (1) for enterprise A profit allocation, the results shown in Table 2. 


\begin{tabular}{|c|c|c|c|c|c|}
\hline $\mathbf{S}$ & $\mathbf{V}(\mathbf{S})$ & $\mathbf{V}(\mathbf{S} \backslash\{\mathbf{i}\})$ & $\mathbf{V}(\mathbf{S}) \ominus \mathbf{V}(\mathbf{S} \backslash\{\mathbf{i}\})$ & $\gamma^{\mathbf{S}}$ & $\gamma^{\mathbf{S}}(\mathbf{V}(\mathbf{S}) \ominus \mathbf{V}(\mathbf{S} \backslash\{\mathbf{i}\}))$ \\
\hline $\mathbf{A}$ & {$[10,14]$} & 0 & {$[10,14]$} & $1 / 3$ & {$[10 / 3,14 / 3]$} \\
\hline$\{\mathbf{A}, \mathbf{B}\}$ & {$[32,40]$} & {$[12,14]$} & {$[20,26]$} & $1 / 6$ & {$[20 / 6,26 / 6]$} \\
\hline$\{\mathbf{A}, \mathbf{C}\}$ & {$[38,44]$} & {$[12,16]$} & {$[26,28]$} & $1 / 6$ & {$[26 / 6,28 / 6]$} \\
\hline$\{\mathbf{A}, \mathbf{B}, \mathbf{C}\}$ & {$[54,60]$} & {$[26,36]$} & {$[28,24]$} & $1 / 3$ & {$[28 / 3,24 / 3]$} \\
\hline
\end{tabular}

Table 2. Enterprise A profit interval based on interval Shapely value

From above table, enterprise A profit interval is $\varphi_{a}(\bar{V})=[20.33,21.67]$. Similarly, $\varphi_{B}(\bar{V})=[15.33,17.67], \varphi_{c}(\bar{V})=[18.33,20.67]$.

\subsection{Post-Improved Profit Allocation with Shapley Interval Value Method}

\subsubsection{Determine the Profit Allocation Indicators and Weights for the Manufacturing and Logistics Industry Alliance}

Based on the characteristics of the manufacturing and logistics industry alliance, this article builds a profit allocation indicator system choosing risk sharing, collaborative effort, marketcompetition, innovative contribution and partner's resource investment as evaluation factors. See table 3.

\begin{tabular}{|c|c|c|}
\hline Goal Layer & Criteria Layer & Indicator Layer \\
\hline \multirow{20}{*}{$\begin{array}{c}\text { profit allocation } \\
\text { indicator system } \\
\text { for manufacturing } \\
\text { and } \\
\text { logistics industry } \\
\text { Alliance C }\end{array}$} & \multirow{4}{*}{$\begin{array}{l}\text { risk sharing } \\
C_{1}\end{array}$} & risk sharing size $C_{11}$ \\
\hline & & risk sharing capacity $C_{12}$ \\
\hline & & risk sharing willingness $C_{13}$ \\
\hline & & probability of failure $C_{14}$ \\
\hline & \multirow{4}{*}{$\begin{array}{l}\text { collaborative efforts } \\
\qquad C_{2}\end{array}$} & willingness to cooperate $C_{21}$ \\
\hline & & system development and execution $\mathrm{C}_{22}$ \\
\hline & & incentive policy development $\mathrm{C}_{23}$ \\
\hline & & organizational culture compatibility $\mathrm{C}_{24}$ \\
\hline & \multirow{4}{*}{$\begin{array}{l}\text { marketcompetition } \\
C_{3}\end{array}$} & marketshare $C_{31}$ \\
\hline & & market growth rate $\mathrm{C}_{32}$ \\
\hline & & profitability $\mathrm{C}_{33}$ \\
\hline & & bargaining power $\mathrm{C}_{34}$ \\
\hline & \multirow{4}{*}{$\begin{array}{c}\text { innovative } \\
\text { contribution } \\
C_{4}\end{array}$} & system innovation $\mathrm{C}_{41}$ \\
\hline & & technical innovation $\mathrm{C}_{42}$ \\
\hline & & cultural innovation $\mathrm{C}_{43}$ \\
\hline & & operational innovation $\mathrm{C}_{44}$ \\
\hline & \multirow{4}{*}{$\begin{array}{c}\text { resource } \\
\text { investment } \\
C_{5}\end{array}$} & hardware investment $C_{51}$ \\
\hline & & software investment $C_{52}$ \\
\hline & & human resources investment $C_{53}$ \\
\hline & & management investment $C_{54}$ \\
\hline
\end{tabular}

Table 3. Profit allocation indicator system for manufacturing and logistics industry alliance 
$\mathrm{W}$, the set of weights is constructed based on the above method. Take the calculation of Criteria Layer as an example. Ten experts are invited to evaluate the five assessment factors in the Criteria Layer to generate an Expert Evaluation Matrix, as shown in Table 4.

\begin{tabular}{|c|c|c|c|c|c|}
\hline expert & $\mathbf{C}_{\mathbf{1}}$ & $\mathbf{C}_{\mathbf{2}}$ & $\mathbf{C}_{\mathbf{3}}$ & $\mathbf{C}_{\mathbf{4}}$ & $\mathbf{C}_{\mathbf{5}}$ \\
\hline $\mathbf{1}$ & 4 & 3 & 3 & 4 & 5 \\
\hline $\mathbf{2}$ & 5 & 2 & 3 & 4 & 4 \\
\hline $\mathbf{3}$ & 3 & 4 & 2 & 3 & 5 \\
\hline $\mathbf{4}$ & 4 & 3 & 3 & 4 & 5 \\
\hline $\mathbf{5}$ & 4 & 3 & 4 & 5 & 5 \\
\hline $\mathbf{6}$ & 3 & 4 & 3 & 5 & 4 \\
\hline $\mathbf{7}$ & 5 & 3 & 2 & 4 & 4 \\
\hline $\mathbf{8}$ & 4 & 4 & 3 & 4 & 5 \\
\hline $\mathbf{9}$ & 4 & 3 & 4 & 5 & 4 \\
\hline $\mathbf{1 0}$ & 4 & 3 & 3 & 4 & 5 \\
\hline
\end{tabular}

Table 4. Expert Evaluation Matrix

get:

$$
\mathrm{F}=\mathrm{X}^{\mathrm{T}} \mathrm{X}=\left[\begin{array}{ccccc}
164 & 125 & 120 & 168 & 183 \\
125 & 106 & 95 & 134 & 148 \\
120 & 95 & 94 & 129 & 138 \\
168 & 134 & 129 & 180 & 192 \\
183 & 148 & 138 & 192 & 214
\end{array}\right]
$$

Using MATLAB to calculate the max eigenvalue of Matrix and get a single root $\rho_{\max }=742.5742$, and its corresponding eigenvector is $X^{*}=\left[\begin{array}{llllll}0.4638 & 0.3706 & 0.3510 & 0.4894 & 0.5337\end{array}\right]$. After Normalization, get the Criteria Layer weight vector, which is $W=\left[\begin{array}{lllll}0.210 & 0.168 & 0.158 & 0.222\end{array}\right.$ 0.242].

Similarly, combination weight of each evaluation indicator in the goal layer can be calculated. Then multiplied by the weight of corresponding evaluation factor in the goal layer to get the combination weight of evaluation indicator in the goal layer respectively, which are,

- Risk sharing weight vector: $W_{1}=\left[\begin{array}{lllll}0.07518 & 0.03024 & 0.04578 & 0.05880\end{array}\right]$.

- Collaborative efforts weight vector: $W_{2}=\left[\begin{array}{llll}0.04519 & 0.05712 & 0.03595 & 0.02974\end{array}\right]$.

- Marketcompetition weight vector: $\mathrm{W}_{3}=\left[\begin{array}{llll}0.0556 & 0.04298 & 0.04076 & 0.0237\end{array}\right]$.

- Innovative contribution weight vector: $\mathrm{W}_{4}=\left[\begin{array}{llll}0.5728 & 0.03685 & 0.03774 & 0.09013\end{array}\right]$.

- Resource investment weight vector: $W_{5}=\left[\begin{array}{lllll}0.06002 & 0.05348 & 0.5493 & 0.07357\end{array}\right]$. 


\subsubsection{Calculate Comprehensive Correction Factor of Profit Allocation}

Ten subject experts are invited to evaluate manufacturing enterprise A. The evaluation standard breaks down into 5 classes, which are high, medium high, medium, medium low and low. The proportions of the number of votes vs. total votes for each class are collected to get the fuzzy comprehensive evaluation matrix for a single factor, shown as follows:

$$
R_{1}=\left[\begin{array}{ccccc}
0.2 & 0.2 & 0.5 & 0.1 & 0 \\
0 & 0.3 & 0.4 & 0.2 & 0.1 \\
0 & 0.2 & 0.4 & 0.4 & 0 \\
0.3 & 0.5 & 0.2 & 0 & 0
\end{array}\right]
$$

Thus, result for enterprise $A$ on risk sharing is $B_{1}=W_{1} \cdot R_{1}=\left[\begin{array}{llllllllll}0.1556 & 0.2984 & 0.3798 & 0.1518\end{array}\right.$ 0.0144]. Similarly, evolution results for other factors such as collaborative efforts, market competition, innovative contribution and resource investment can also be calculated. The criteria layer comprehensive evaluation matrix is:

$$
R=\left[\begin{array}{ccccc}
0.1556 & 0.2984 & 0.3798 & 0.1518 & 0.0144 \\
0.0947 & 0.6746 & 0.1468 & 0.0661 & 0.0447 \\
0.109 & 0.1972 & 0.372 & 0.1896 & 0.0272 \\
0.0686 & 0.6428 & 0.1742 & 0.2472 & 0.0166 \\
0.1961 & 0.1794 & 0.259 & 0.1433 & 0.2222
\end{array}\right]
$$

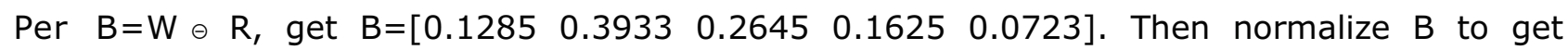
$B^{\prime}=\left[\begin{array}{lllll}0.126 & 0.385 & 0.259 & 0.159 & 0.071\end{array}\right]$, then multiply $B^{\prime}$ and fuzzy evaluation vector $V$ to get enterprise $A^{\prime} s$ comprehensive impact factor of $P_{A}=B^{\prime} V^{\top}=0.5672$, where fuzzy evaluation vector $V=\left[\begin{array}{lllll}0.9 & 0.7 & 0.5 & 0.3 & 0.1\end{array}\right]$. Equally, for enterprise $B$ and $C, P_{B}=0.7755, P_{C}=0.8154$. After normalization, the $A, B, C$ profit allocation comprehensive impact factors are $P_{A}^{\prime}=0.2628$, $P_{B}{ }^{\prime}=0.3594$ and $P_{C}{ }^{\prime}=0.3778$ respectively. Therefore the correction factors for each enterprise are $\Delta P_{A}=P_{A}^{\prime}-1 / 3=-0.0705, D P_{B}=P_{B}^{\prime}-1 / 3=0.026, \Delta P_{C}=P_{C}^{\prime}-1 / 3=0.0445$.

It is worth nothing that the dynamic nature of alliance development and the uncertainty in alliance operation lead to the variation of factor weights. Under different circumstances, such as in the different stages of alliance development, evaluation for specific factors may fluctuate. 


\subsubsection{Calculate Improved Profit Allocation Interval}

Using formula (2) to get enterprise A, B, C alliance improved actual profit allocation interval: $\varphi_{\mathrm{A}}^{\prime}(\bar{V})=[16.523,17.44], \varphi_{\mathrm{B}}^{\prime}(\bar{V})=[16.734,19.23], \varphi_{\mathrm{C}}(\bar{V})=[20.733,23.34]$.

From the above results, after taking into consideration of the risk sharing, collaborative efforts, market competition, innovative contribution and resource investment, profit allocation interval for the enterprise $A, B$ and $C$ has been re-adjusted. Profit interval of manufacturing enterprise $A$ has been reduced, while logistics enterprises $B$ and $C$ profit interval has been increased. The interval Shapley Value method with comprehensive correction factor is more scientific and reasonable, and more in line with the actual situation.

\section{Conclusions}

Profit allocation between manufacturing and logistics alliance is a complicated issue. In reality, the alliance partners are often unclear on the expected return or the profit allocation under different alliance strategy. In addition, during the process of cooperation, many factors affect the profit allocation and different factors have different importance. This article, based on manufacturing and logistics industry alliance characteristics, used AHP-GEM method, an indicator system is established for profit allocation in manufacturing and logistics industry alliance. Five impact factors are identified for the alliance, which are risk sharing, collaborative efforts, market competition, innovative contribution and partner resources investment. Comprehensive impact factors are calculated in profit allocation of each partner using fuzzy evaluation method. Correction factor is introduced to improve interval Shapley Value method. An improved model for profit allocation is established for fuzzy cooperative games between manufacturing and logistics industries. Improved interval Shapley value method is more logical and reasonable, which has practical value in solving the profit allocation issues of manufacturing and logistics industry alliance.

\section{Current projects}

Anhui Provincial College Level Humanities and Social Sciences Key Research Project "The manufacturing and logistics industry cooperative game study in low-carbon economy (SK2012A106)"; China Institute of Logistics research project No. 2012CSLKT105. 


\section{References}

Chen, Q.Q., Li, B. (2011). Study on Decision-making and Incentive Contract Design for Relationship-oriented Investment in Logistics Outsourcing. LogisticsTechnology, 7(30), 101104.

Chen, W., Zhang, Q. (2006). Shapley Value for Fuzzy Cooperative Games. Journal of Management Science in China, 5(9), 50-55.

Luo, T.J., Mao, K.Y. (2010). Game Analysis on Pricing of Logistics Outsourcing. China Business \& Trade, 6(20), 107-188.

Mares, M. (2001). Fuzzy Cooperative Games: Cooperation with Vague Expectations (pages 100-118). New York: Physica-Verlag. http://dx.doi.org/10.1007/978-3-7908-1820-8

Meng, F.Y., Zhang, Q. (2011). The Shapley Function for Fuzzy Cooperative Games with Interval Payoffs. Transactions of Beijing Institute of Technology, 9(31), 1131-1134.

Ni, L.L. (2007). Game Analysis of Logistics Outsourcing and Study on Risk Evasion Tactics. Logistics Technology, 11(26), 125-127.

Qiu, W.H. (1997). An Eigenvalue Method on Group Decision. Applied Mathematics and Mechanics, 11(18), 1027-1031.

Surajit, B. (2008). Cooperative Games with Fuzzy Coalitions and Fuzzy Characteristic Functions. Fuzzy Set and Systems, 159, 138-151. http://dx.doi.org/10.1016/j.fss.2007.07.007

Tan, C.Q. (2010). Shapley Value for n-person Games with Interval Coalition Worth. Acta Mathematicae Applicatae Sinica, 2(33), 193-203.

Tan, C.Q. (2011). Fuzzy Shapley Value for n-Person Games with Fuzzy Coalition Value. Journal of Systems \& Management, 1(21), 42-47.

Yu, X.H., Zhang, Q.(2008). Profit Allocation in Production Cooperative Game Based on Interval Shapley Value. Transactions of Beijing Institute of Technology, 7(28), 655-658.

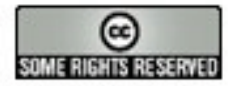

Article's contents are provided on a Attribution-Non Commercial 3.0 Creative commons license. Readers are allowed to copy, distribute and communicate article's contents, provided the author's and Journal of Industrial Engineering and Management's names are included. It must not be used for commercial purposes. To see the complete license contents, please visit http://creativecommons.org/licenses/by-nc/3.0/. 